Article

\title{
Simultaneous Determination of Multiple Classes of Hydrophilic and Lipophilic Components in Shuang-Huang-Lian Oral Liquid Formulations by UPLC-Triple Quadrupole Linear Ion Trap Mass Spectrometry
}

\author{
Jun Liang *, Hui-Min Sun and Tian-Long Wang \\ Key Laboratory of Chinese MateriaMedica, Heilongjiang University of Chinese Medicine, Ministry of Education, \\ Harbin 150040, China; hhuiminsun@163.com (H.-M.S.); t1890322@163.com (T.-L.W.) \\ * Correspondence: lliangjunn@163.com; Tel.: +86-451-8219-5301
}

Received: 25 October 2017; Accepted: 23 November 2017; Published: 24 November 2017

\begin{abstract}
The Shuang-Huang-Lian (SHL) oral liquid is a combined herbal prescription used in the treatment of acute upper respiratory tract infection, acute bronchitis and pneumonia. Multiple constituents are considered to be responsible for the therapeutic effects of SHL. However, the quantitation of the multi-components from multiple classes is still unsatisfactory because of the high complexity of constituents in SHL. In this study, an accurate, rapid, and specific UPLC-MS/MS method was established for simultaneous quantification of 18 compounds from multiple classes in SHL oral liquid formulations. Chromatographic separation was performed on a HSS T3 $(1.8 \mu \mathrm{m}, 2.1 \mathrm{~mm} \times 100 \mathrm{~mm})$ column, using a gradient mobile phase system of $0.1 \%$ formic acid in acetonitrile and $0.1 \%$ formic acid in water at a flow rate of $0.2 \mathrm{~mL} \cdot \mathrm{min}^{-1}$; the run time was $23 \mathrm{~min}$. The MS was operated in negative electrospray ionization (ESI ${ }^{-}$) for analysis of 18 compounds using multiple reaction monitoring (MRM) mode. UPLC-ESI ${ }^{-}$-MRM-MS/MS method showed good linear relationships $\left(R^{2}>0.999\right)$, repeatability (RSD $<3 \%$ ), precisions (RSD $<3 \%$ ) and recovery (84.03-101.62\%). The validated method was successfully used to determine multiple classes of hydrophilic and lipophilic components in the SHL oral liquids. Finally, principal component analysis (PCA) was used to classify and differentiate SHL oral liquid samples attributed to different manufacturers of China. The proposed UPLC-ESI ${ }^{-}$-MRM-MS/MS coupled with PCA has been elucidated to be a simple and reliable method for quality evaluation of SHL oral liquids.
\end{abstract}

Keywords: UPLC-MS/MS; Shuang-Huang-Lian; multi-ingredient quantitative analysis; quality control; principal component analysis

\section{Introduction}

Shuang-Huang-Lian (SHL) is a famous Chinese formula prepared from three Traditional Chinese Medicines (TCMs) including Lonicera Japonica (Jinyinhua), Radix Scutellariae (Huangqin) and Fructus Forsythiae (Lianqiao). SHL oral liquid has been widely applied to be effective clinical therapeutics for the treatment of acute upper respiratory, tract infection, acute bronchitis and pneumonia [1,2]. Flavonoids, lignans and phenylpropanoids have been confirmed to be the main and effective components in SHL oral liquid [1,3]. These ingredients were proven to be responsible for the various biological activities of this Chinese formula [1-4]. Many of these natural products displayed a wide range of biological activities such as antibacterial, antioxidant, anti-inflammatory activities. So quantification of these compounds would be of great significance to guarantee good quality of this formula of SHL. 
Although quantitative analysis of single and a few compounds in this formula has been reported by capillary electrophoresis with electrochemical detection (CE-ECD) [5], high performance liquid chromatography with diode array detection (HPLC-DAD) [6], HPLC with mass spectrometry (HPLC-MS) [7], HPLC with evaporative light scattering detection (HPLC-ELSD) [8], and HPLC-DAD-ECD [9], a simultaneous analysis of flavonoids, lignans and phenylpropanoids in this prescription is still missing through UPLC-ESI-MS/MS based on multiple reaction monitoring (MRM) for speedy quality control.

UPLC is based on available reverse phase chromatographic media with a $1.7 \mu \mathrm{m}$ particle size, together with a liquid system that can be operate such columns at much higher pressures. In comparison with common HPLC, UPLC offers many advantages including higher separation efficiency, shorter analysis time and less solvent consumption. Furthermore, UPLC hyphenated MS technique offers the possibility to obtain a more comprehensive chemical profiles and quantization by utilizing different ion modes and high sensitivity $[10,11]$.

In this study, a simple, accurate and sensitive method based on UPLC-ESI ${ }^{-}$-MRM-MS/MS was developed for simultaneous determination of multiple hydrophilic and lipophilic components from multiple classes attributed to two quinic acids ( 1 and 11$)$, one phenylpropionic acid (2), three phenylethanoid glycosides $(3,4$ and 7$)$, eight flavonoids $(5,6,8,9,12,13,17$ and 18), four lignans (10 and 14-16). These compounds were reported in SHL oral liquids, including chlorogenic acid (1), caffeic acid (2), lianqiaoxinside A (3), forsythiaside B (4), rutin (5), hyperoside (6), forsythiaside A (7), cynaroside (8), scutellarin (9), (+)-pinoresinol- $\beta$-D-glucoside (10), isochlorogenic acid A (11), baicalein (12), baicalin (13), phillyrin (14), phillygenin (15), arctiin (16), quercetin (17) and luteolin (18). The current study provided a further refinement of the methods for the quality control of this traditional prescription.

\section{Results and Discussion}

\subsection{Optimization of UPLC-MS/MS Conditions}

Three reversed-phase chromatographic columns, including ACQUITY UPLC HSS T3 $(1.8 \mu \mathrm{m}$, $2.1 \mathrm{~mm} \times 100 \mathrm{~mm})$, ACQUITY UPLC BEH C $18(1.7 \mu \mathrm{m}, 2.1 \mathrm{~mm} \times 100 \mathrm{~mm})$ and Cortecs UPLC $\mathrm{C}_{18}(1.6 \mu \mathrm{m}, 2.1 \mathrm{~mm} \times 100 \mathrm{~mm})$ were tested with the same sample solution. The results showed that the HSS T3 column displayed acceptable separation capacity. ACN $/ \mathrm{H}_{2} \mathrm{O}$ system provided the best performance through the optimization of different mobile phases $\left(\mathrm{MeOH} / \mathrm{H}_{2} \mathrm{O}, \mathrm{ACN} / \mathrm{H}_{2} \mathrm{O}\right.$ and $\mathrm{ACN} / \mathrm{MeOH} / \mathrm{H}_{2} \mathrm{O}$ ). Several different modifiers were investigated (none, formic acid, and ammonium formate), and the results showed that formic acid provided the best peak shape. Additional UPLC conditions were optimized by varying column temperatures $\left(25,30,35\right.$ and $\left.40^{\circ} \mathrm{C}\right)$, and flow rates $(0.2$, 0.3 and $0.40 \mathrm{~mL} / \mathrm{min}$ ). The optimized UPLC conditions provided the highest selectivity and resolution. These were: ACQUITY UPLC HSS T3 column at $35{ }^{\circ} \mathrm{C}, 0.1 \%$ formic acid in water (A) and $0.1 \%$ formic acid in acetonitrile (B) mobile phase gradient at a flow rate of $0.20 \mathrm{~mL} / \mathrm{min}$.

Under the chosen chromatographic conditions, compounds (1-18) showed to be present of the more intensive $[\mathrm{M}-\mathrm{H}]^{-}$ions in the negative ion mode than those of $[\mathrm{M}+\mathrm{H}]^{+}$or $[\mathrm{M}+\mathrm{Na}]^{+}$in the positive mode. Thus, a 4000 QTRAP UPLC-MS/MS system equipped with ESI interface in negative mode was used for detection in MRM mode. As shown in Figure 1, reference standards (1-18) showed good peak shapes and excellent resolutions. The main MS parameters including declustering potential (DP), collision energy (CE) were acquired and summarized in Table 1.

The major fragmentation pathways of $\mathbf{1}-\mathbf{1 8}$ were also clarified in Figure 1. Compounds $\mathbf{1}$ and 11 afforded major product ion at $m / z 191.0$ due to the preferential cleavage of ester glycosidic bonds. A neutral loss of $\mathrm{CO}_{2}$ was readily observed for compound 2 to produce typical product ion at $\mathrm{m} / \mathrm{z}$ 135.2. The cleavage of ester glycosidic bonds was observed for compounds 3,4 and 7 due to the present of the caffeoyl moieties. The compounds 5, 6, 8, 9, 13, 14 and 16 underwent dissociation of monosaccharide glycosidic bonds to give the corresponding product ions at $m / z 300.9,299.9,285.0$, 
284.9, 371.0 and 371.2, respectively. The characteristic product ion at $m / z 150.9$ for compound 10 was formed by cross-ring cleavages of tetrahydrofuran rings. Three flavonoids 12, 17 and 18 experienced the retro-Diels-Alder fragmentation reaction of C-ring opening and B-ring cleavage to afford the corresponding product ions at $m / z 138.7,151.2$ and 133.1, respectively. Therefore, the MRM transition used those intact deprotonated $m / z$ values of precursors $\left(Q_{1}\right)$ and major fragments as product ions $\left(\mathrm{Q}_{3}\right)$ for accurate detection of these compounds 1-18.

Table 1. Main MS parameters for UPLC-MS/MS analysis.

\begin{tabular}{|c|c|c|c|c|c|c|c|c|}
\hline No. & Compound Name & $\begin{array}{l}\text { Retention } \\
\text { Time (min) }\end{array}$ & $\begin{array}{c}\text { Molecular } \\
\text { Weight }\end{array}$ & $\begin{array}{l}\text { Selected Ion } \\
(\mathrm{m} / \mathrm{z})\end{array}$ & $\mathrm{Q}_{1}$ & $\mathrm{Q}_{3}$ & DP & $\mathrm{CE}$ \\
\hline 1 & Chlorogenic acid & 3.40 & 354.31 & {$[\mathrm{M}-\mathrm{H}]^{-}$} & 352.90 & 191.00 & -59.56 & -19.70 \\
\hline 3 & Lianqiaoxinside A & 9.09 & 624.59 & {$[\mathrm{M}-\mathrm{H}]^{-}$} & 623.00 & 161.00 & -157.08 & -57.85 \\
\hline 4 & Forsythiaside B & 9.49 & 756.70 & {$[\mathrm{M}-\mathrm{H}]^{-}$} & 755.00 & 593.60 & -160.00 & -50.93 \\
\hline 5 & Rutin & 9.61 & 610.51 & {$[\mathrm{M}-\mathrm{H}]^{-}$} & 609.30 & 300.90 & -163.89 & -47.96 \\
\hline 8 & Cynaroside & 10.14 & 448.38 & {$[\mathrm{M}-\mathrm{H}]^{-}$} & 447.00 & 285.00 & -138.97 & -39.83 \\
\hline 9 & Scutellarin & 10.19 & 462.37 & {$[\mathrm{M}-\mathrm{H}]^{-}$} & 461.00 & 284.90 & -78.92 & -26.24 \\
\hline 10 & (+)-Pinoresinol- $\beta$-D-glucoside & 11.57 & 520.53 & {$[\mathrm{M}-\mathrm{H}]^{-}$} & 519.00 & 150.90 & -84.64 & -44.99 \\
\hline 11 & Isochlorogenic acid $\mathrm{A}$ & 12.09 & 516.45 & {$[\mathrm{M}-\mathrm{H}]^{-}$} & 515.00 & 191.20 & -75.30 & -44.90 \\
\hline 12 & Baicalein & 17.47 & 270.24 & {$[\mathrm{M}-\mathrm{H}]^{-}$} & 269.00 & 138.70 & -100.31 & -42.71 \\
\hline 13 & Baicalin & 17.50 & 446.37 & {$[\mathrm{M}-\mathrm{H}]^{-}$} & 444.80 & 269.20 & -89.57 & -26.28 \\
\hline 17 & Quercetin & 21.89 & 302.24 & {$[\mathrm{M}-\mathrm{H}]^{-}$} & 301.10 & 151.20 & -88.89 & -30.26 \\
\hline 18 & Luteolin & 21.91 & 286.24 & {$[\mathrm{M}-\mathrm{H}]^{-}$} & 285.00 & 133.10 & -106.49 & -47.79 \\
\hline
\end{tabular}
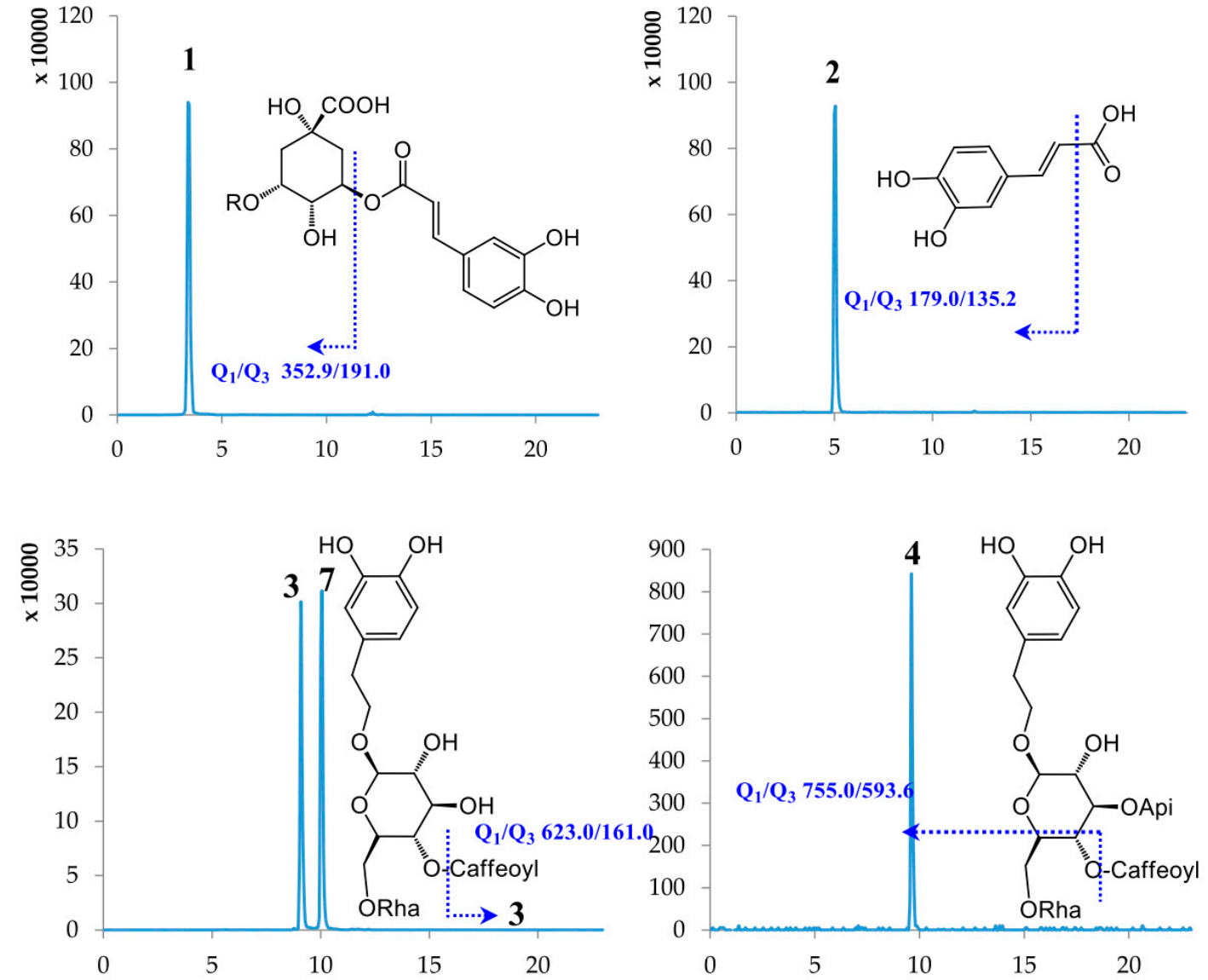

Figure 1. Cont. 

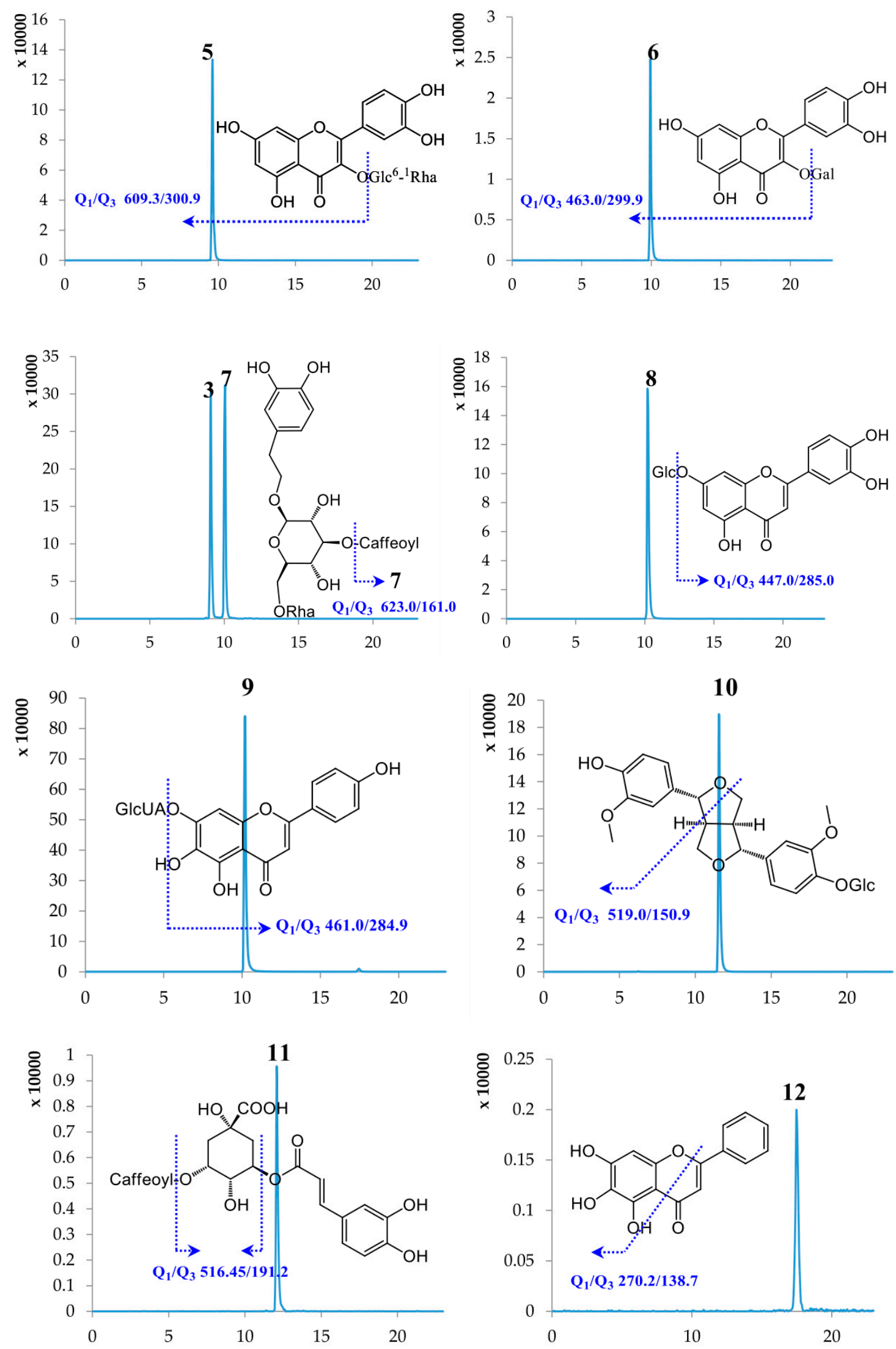

Figure 1. Cont. 

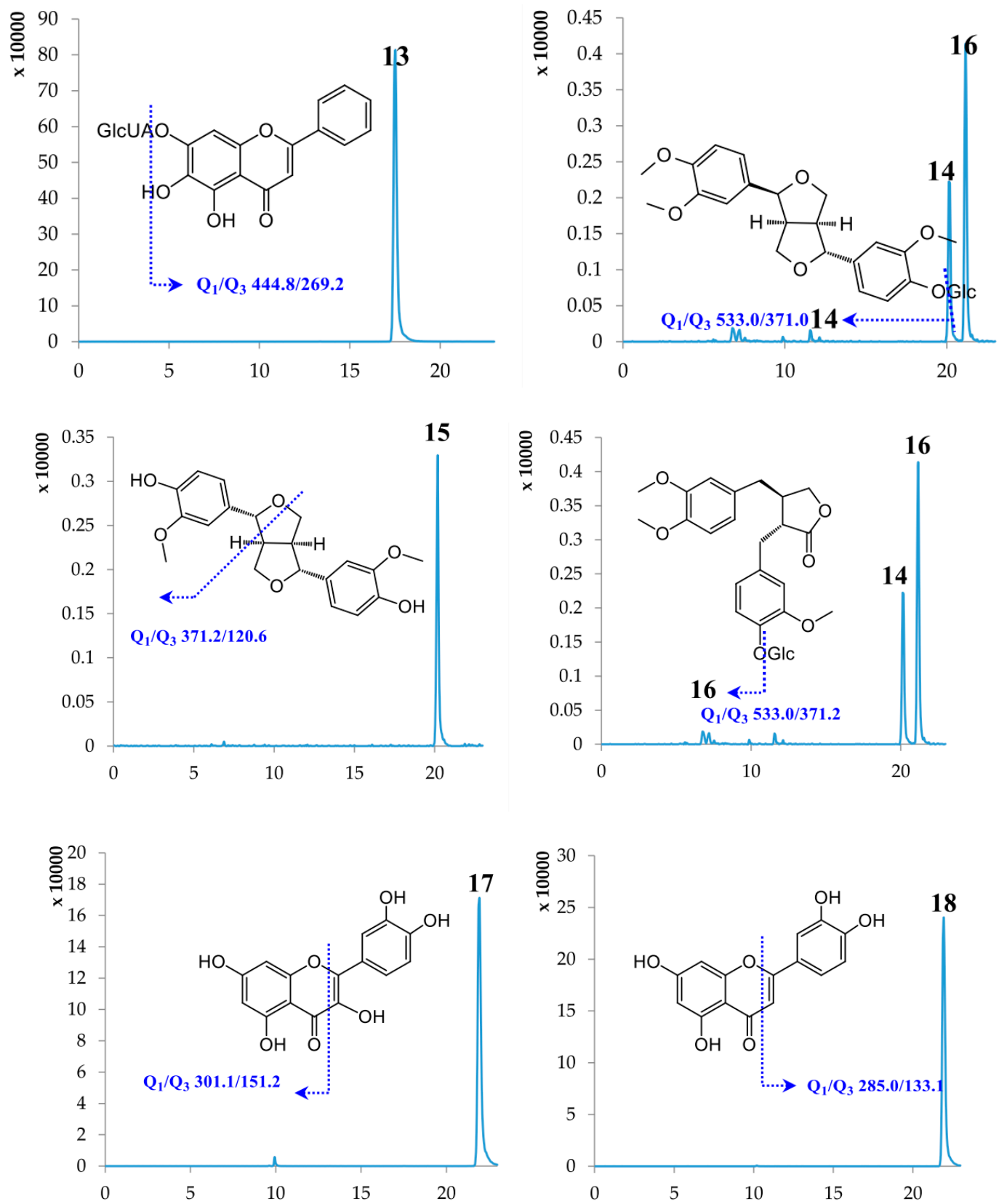

Figure 1. MRM chromatograms and their major fragmentations of reference standards 1-18.

\subsection{Method Validation}

The results of calibration were summarized in Table 2 and good correlations were found between the peak area $(y)$ and concentration of tested compounds $(x)(r>0.999)$ within test ranges. The limit of detections (LODs) and the limit of quantifications (LOQs) for all standard analytes were in the range of $2.44-78.13 \mathrm{ng} / \mathrm{mL}$ and $4.88-156.25 \mathrm{ng} / \mathrm{mL}$, respectively, indicating that this method is sensitive for the quantitative determination of major components in SHL oral liquid samples.

Repeatability of this method was obtained by analyzing six different samples using the same preparation procedure. RSD values of component content and retention time of these 18 compounds were all less than $3.0 \%$, which satisfied the criteria of quantitative analysis.

Intra-day and inter-day variability was used to evaluate precision. Six sample solutions respectively prepared as described above and mixed standard solutions of eighteen compounds at low, medium and high concentrations on 1 day $(n=6)$ and on three consecutive days, were analyzed, 
respectively. The results indicated that the mean intra-day and inter-day RSD were less than 3.0\%. Results from determination of intra-day and inter-day precision (as RSD) are shown in Table S1.

Table 2. Summarization of calibration results, LOD and LOQ values.

\begin{tabular}{cccccc}
\hline No. & Regression Equation & Linear Range $(\mu \mathbf{g} / \mathbf{m L})$ & $\boldsymbol{R}^{\mathbf{2}}$ & LODs $(\mathbf{n g} / \mathbf{m L})$ & LOQs $(\mathbf{n g} / \mathbf{m L})$ \\
\hline $\mathbf{1}$ & $y=4 \times 10^{6} x-32,641$ & $0.02-5.00$ & 0.9993 & 4.88 & 9.76 \\
$\mathbf{2}$ & $y=4 \times 10^{6} x+52,653$ & $0.02-5.00$ & 0.9990 & 4.88 & 9.76 \\
$\mathbf{3}$ & $y=1 \times 10^{6} x-63,209$ & $0.02-5.00$ & 0.9992 & 4.88 & 9.76 \\
$\mathbf{4}$ & $y=2482.3 x-626.91$ & $0.31-10.00$ & 0.9997 & 78.13 & 156.25 \\
$\mathbf{5}$ & $y=375,597 x-14,725$ & $0.02-2.50$ & 0.9992 & 4.88 & 9.76 \\
$\mathbf{6}$ & $y=78,413 x-2751.8$ & $0.02-5.00$ & 0.9999 & 9.76 & 19.53 \\
$\mathbf{7}$ & $y=1 \times 10^{6} x-88,370$ & $0.02-10.00$ & 0.9992 & 4.88 & 9.76 \\
$\mathbf{8}$ & $y=604,060 x+9178.1$ & $0.04-10.00$ & 0.9999 & 2.44 & 4.88 \\
$\mathbf{9}$ & $y=3 \times 10^{6} x-38,112$ & $0.02-5.00$ & 0.9995 & 4.88 & 9.76 \\
$\mathbf{1 0}$ & $y=708,705 x-58,709$ & $0.02-10.00$ & 0.9993 & 2.44 & 4.88 \\
$\mathbf{1 1}$ & $y=38,443 x-1932.4$ & $0.02-5.00$ & 0.9995 & 4.88 & 19.53 \\
$\mathbf{1 2}$ & $y=13,450 x+85.951$ & $0.08-10.00$ & 0.9999 & 4.88 & 19.53 \\
$\mathbf{1 3}$ & $y=1 \times 10^{6} x+524,990$ & $0.63-50.00$ & 0.9991 & 19.53 & 78.13 \\
$\mathbf{1 4}$ & $y=10,777 x-216.5$ & $0.04-10.00$ & 0.9997 & 2.44 & 9.77 \\
$\mathbf{1 5}$ & $y=13,664 x-918.63$ & $0.08-10.00$ & 0.9998 & 9.77 & 39.06 \\
$\mathbf{1 6}$ & $y=21,338 x-1861.1$ & $0.04-10.00$ & 0.9995 & 9.77 & 39.06 \\
$\mathbf{1 7}$ & $y=1 \times 10^{6} x+5883.7$ & $0.02-10.00$ & 0.9993 & 2.44 & 9.77 \\
$\mathbf{1 8}$ & $y=1 \times 10^{6} x-3265.6$ & $0.02-10.00$ & 0.9997 & 2.44 & 9.77 \\
\hline
\end{tabular}

$y$ is the peak areas of reference standards, and $x$ is the value of the reference compound's concentration $(\mu \mathrm{g} / \mathrm{mL})$.

For the stability test, retention time and peak area of eighteen compounds in sample solution were analyzed in $0,2,4,8,16,32$ and $48 \mathrm{~h}$. RSD values of the retention time and peak area of eighteen compounds were less than $0.5 \%$ and $3.0 \%$, respectively. These results suggested that it was feasible to analyze samples within 2 days.

The accuracy of the method was validated by measuring recovery through standard addition method. A known amount (low, medium and high) of the eighteen standard references were spiked into samples. Quantity of each component was subsequently obtained by use of the corresponding calibration plots. Each set of samples was analyzed three times. The RSD values were in the range of $1.17-4.78 \%$ and recoveries of analytes varied from $84.03 \%$ to $101.62 \%$. Above results exhibited the reliability and accuracy for the measurement of these constituents. The recovery was calculated as follows: recovery $(\%)=100 \times($ amount found - original amount)/amount spiked, as shown in Table S2.

\subsection{Sample Analysis}

The validated method was successfully applied for the identification and quantification of 18 active compounds in 14 batches of SHL oral liquids. Regarding as these 18 compounds due to multiple hydrophilic and lipophilic components from multiple classes, there were two quinic acids (1 and 11), one phenylpropionic acid (2), three phenylethanoid glycosides (3, 4 and 7), eight flavonoids (5, 6, 8, 9, 12, 13, 17 and 18), four lignans (10 and 14-16). Meanwhile, these 18 constituents were classified into three groups according to their source of raw materials. It was characterized by eight active compounds 1, 2, 5, 6, 8, 11, 17 and 18 from Jinyinhua [12], four compounds 9, 12, 13 and 18 from Huangqin [13], and nine compounds 3-5, 7, 10 and 14-17 from Lianqiao [14]. Although three compounds 5, 17 and 18 were simultaneously observed in two raw materials, other fifteen ingredients covered major and specific ones from different species. Also, this is the first report on simultaneous determination of these hydrophilic and lipophilic components in SHL oral liquid formulations by single LC-MS/MS run based on MRM mode. The contents of the investigated compounds (Figure 2), based on their respective calibration curves, are summarized in Table 3. Among these compounds, baicain (13) was 
found to be the most dominant constituents in all samples tested, at amounts of $12.27-15.90 \mathrm{mg} / \mathrm{g}$. In addition, chlorogenic acid (1) and forsythiaside A (7) were also very abundant in 14 batches of SHL oral liquid. On the contrary, three compounds, forsythiaside B (4), arctiin (16) and quercetin (17), were not detected at all. This may be explained by specific processing method of SHL oral liquids.

Obvious differences could be further observed through performing principal component analysis (PCA) in Figure 3. PCA is a useful tool of chemometrician for data compression and information extraction which find combinations of variables or factors that describe major trends in a data set. It was noticeable that these 14 samples tested apparently form into four groups a-d according to their manufacturers (Figure 3A). The groups a-d were assigned to be Sanjing, Tailong, Zhenbaodao and Baitian'e, respectively. The corresponding PCA loading plot is illustrated in Figure 3B. Clearly, chlorogenic acid (1), lianqiaoxinside A (3), (+)-pinoresinol- $\beta$-D-glucoside (10), and phillygenin (15) were found at higher amounts in samples 7 and 8 from Tailong but caffeic acid (2) was lower in samples 7 and 8 compared with samples 9, 10 and 11 from Zhenbaodao. Thus, the concentration of some analytes varied greatly among the different samples, which was probably due to growing condition, climate, and drug processing of crude herbs. So, detection of a single or only several components could not effectively guarantee the quality of SHL oral liquids. It is essential for carry out simultaneous determination of multiple gradients for quality control of the herbal prescription.
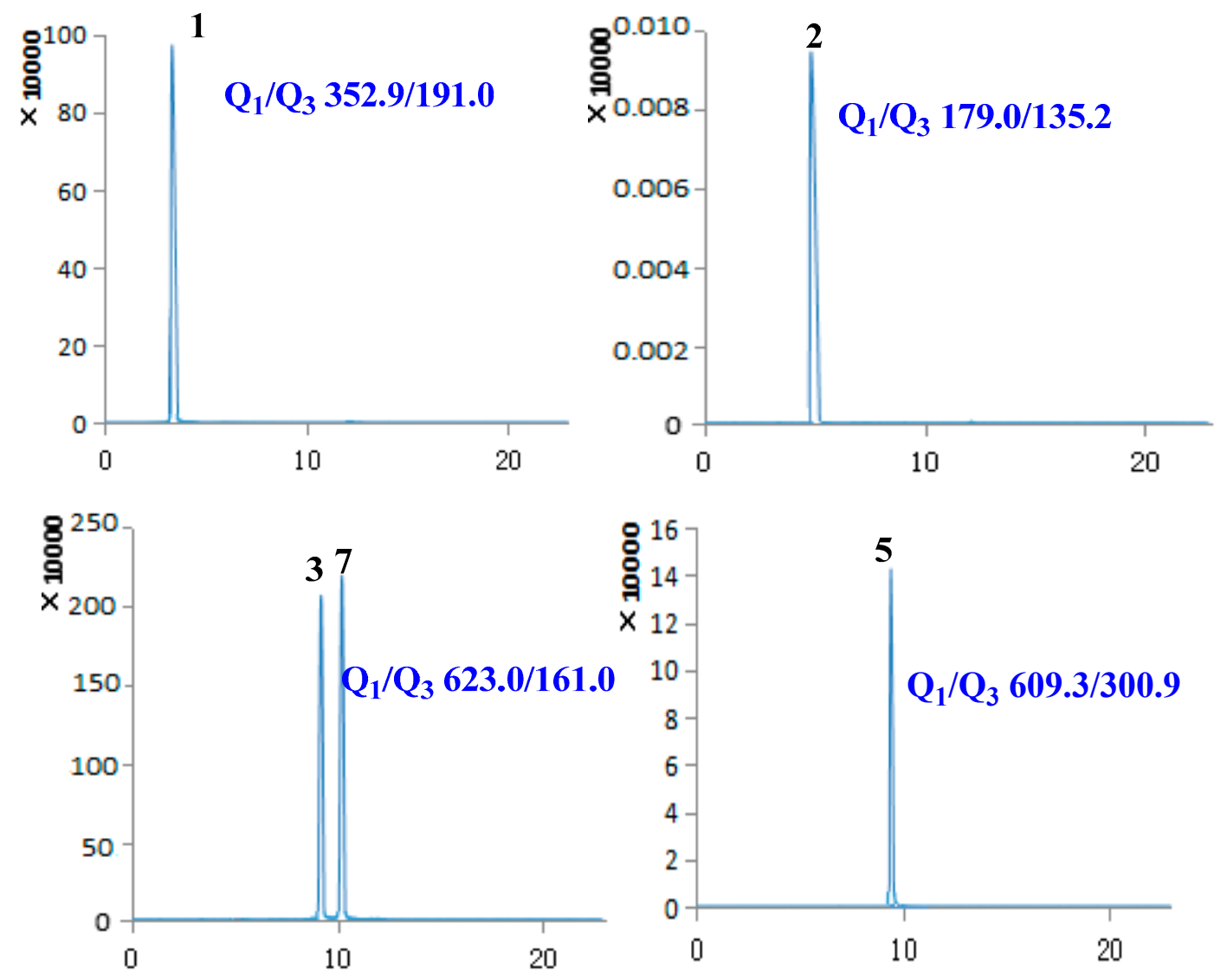

Figure 2. Cont. 

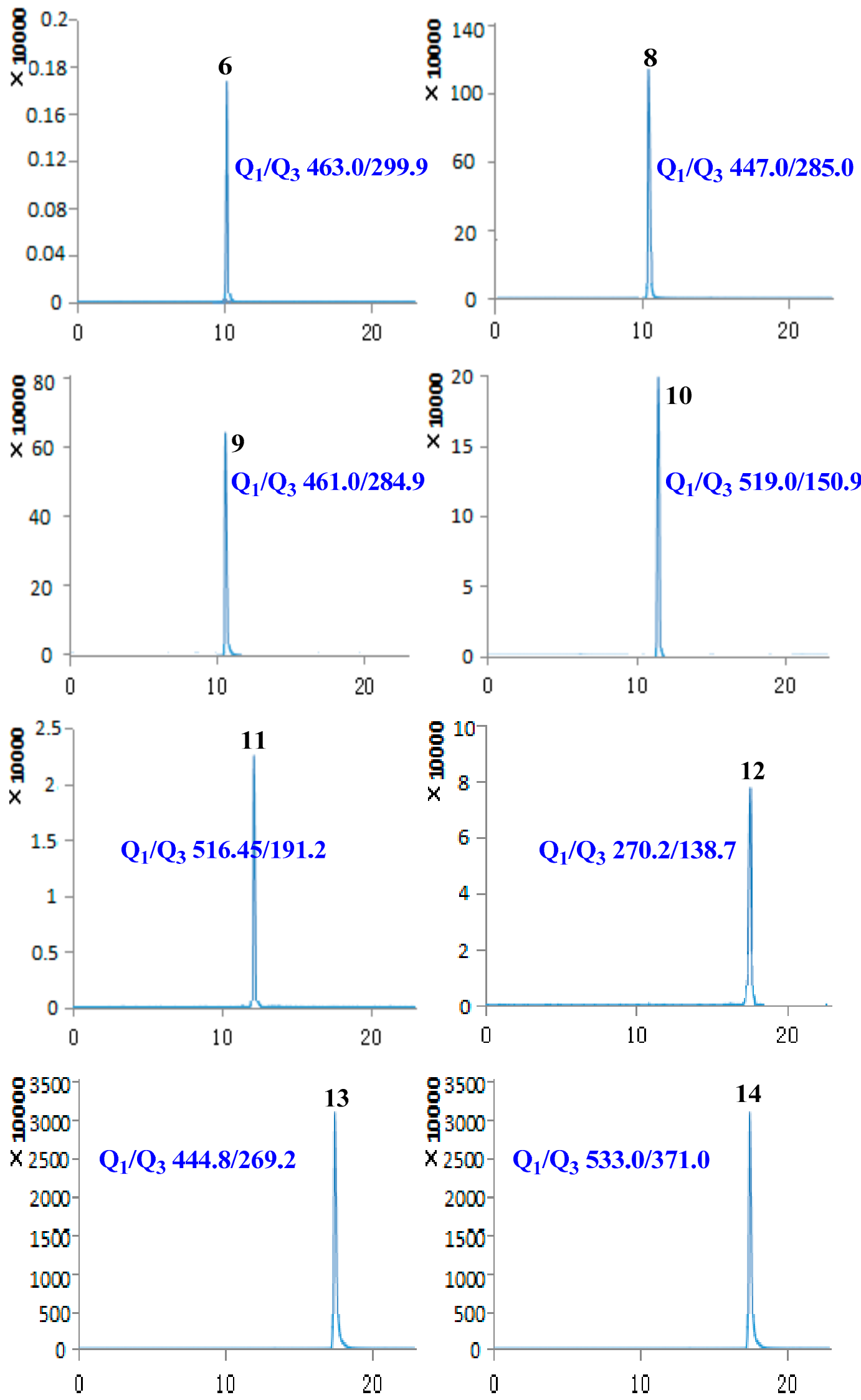

Figure 2. Cont. 

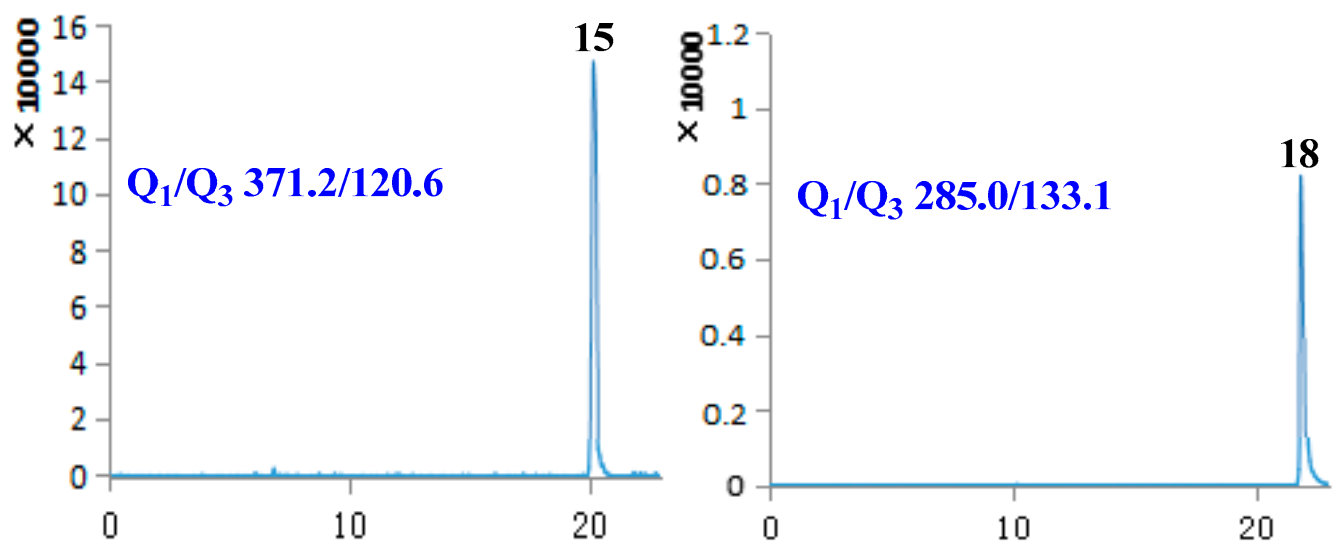

Figure 2. MRM chromatograms of 1-3, 5-15 and 18 in sample.

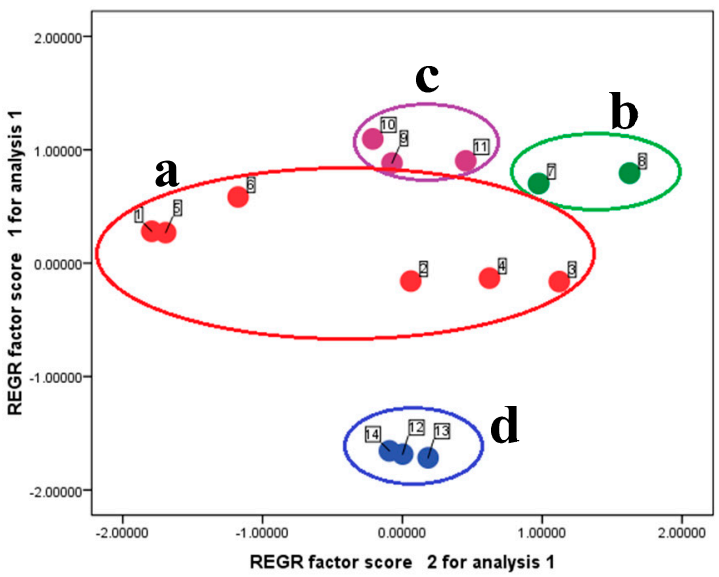

(A)

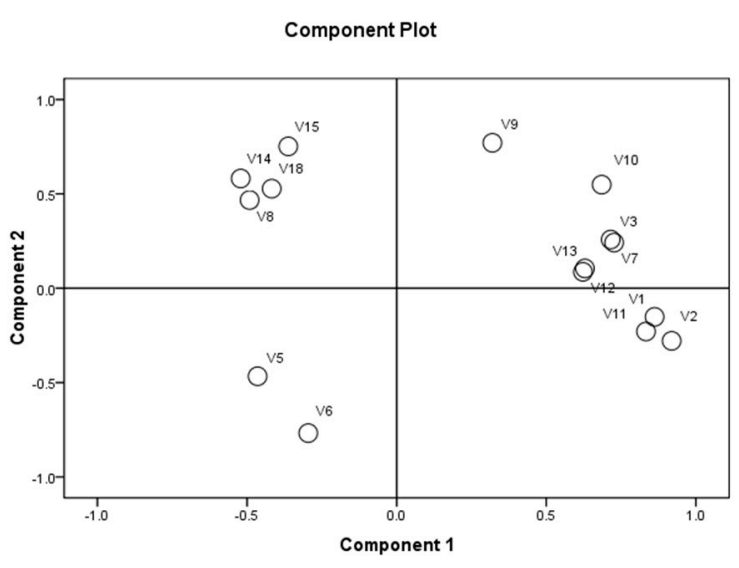

(B)

Figure 3. (A) Score plots from PCA; (B) loading plots from PCA. 
Table 3. Contents $(\mathrm{mg} / \mathrm{g}$ ) of main constituents in 14 different batches of SHL oral liquids.

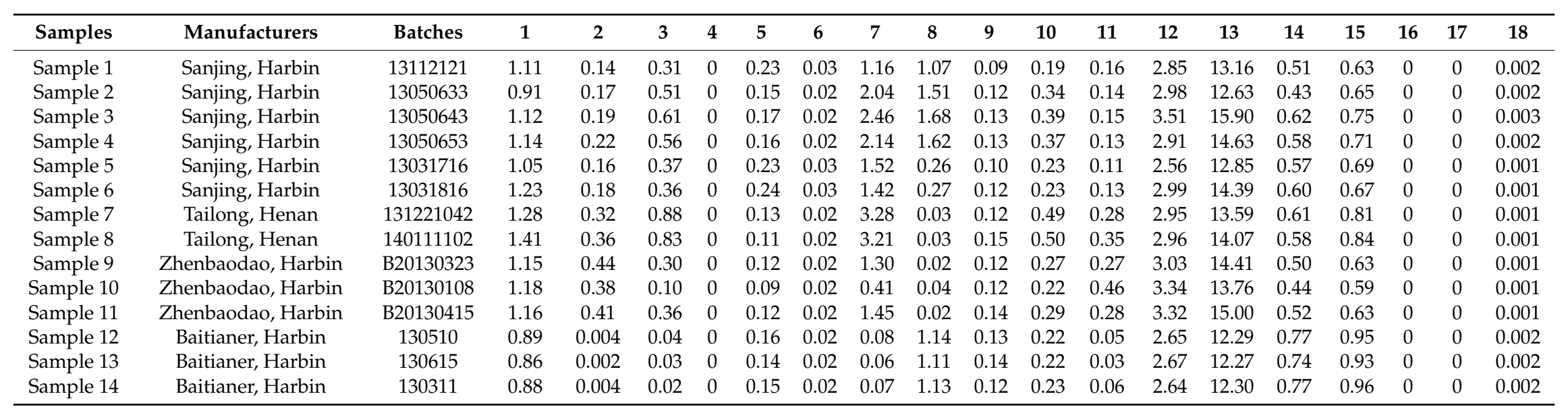




\section{Materials and Methods}

\subsection{Chemicals and Materials}

Lianqiaoxinside A was isolated by the author from the fruits of F. suspensa. Chlorogenicacid, caffeic acid, forsythiaside B, rutin, hyperoside, forsythiaside A, cynaroside, scutellarin, (+)-pinoresinol- $\beta$-D-glucoside, isochlorogenic acid A, baicalein, baicalin, phillyrin, phillygenin, arctiin, quercetin, luteolin $(\geq 98.0 \%)$ were purchased from the Chengdu MUST Biotechnology Co., Ltd. (Chengdu, China). HPLC grade acetonitrile (ACN) and formic acid were obtained from Fisher Scientific (Waltham, MA, USA). Purified water was used from a Milli-Q system (Millipore, Bedford, MA, USA). All other reagents were of analytical grade.

\subsection{Preparation of Standard Solutions}

The standards for Chlorogenic acid, caffeic acid, lianqiaoxinside A, forsythiaside B, rutin, hyperoside, forsythiaside A, cynaroside, scutellarin, (+)-pinoresinol- $\beta$-D-glucoside, isochlorogenic acid A, baicalein, baicalin, phillyrin, phillygenin, arctiin, quercetin, luteolin were weighed accurately and dissolved in methanol at a concentration of $1 \mathrm{mg} \mathrm{mL}^{-1}$. A mixed intermediate stock standard solution was then prepared in methanol; the concentrations of compounds in this solution were $10 \mathrm{ug} \cdot \mathrm{mL}^{-1}$ except that baicalin was $40 \mathrm{ug} \cdot \mathrm{mL}^{-1}$. The stock solutionsforeach quantitative analytes were further diluted with methanol to achieve a series of working solutions used to establish the calibration curves. The standard stock solutions and the working standard solutions were stored in brown vials at $4{ }^{\circ} \mathrm{C}$.

\subsection{Samples Preparation}

14 batches of SHL oral liquid were collected from different manufacturers. Commercial product SHL 1-6 (lot No. 13112121, 13050633, 13050643, 13050653, 13031716, 13031816), SHL 7-8 (lot No. 131221042, 140111102), SHL 9-11 (lot No. B20130323, B20130108, B20130415) and SHL 12-14 (lot No. 130510, 130617, 130626) were purchased from Sanjing Pharmaceutical Co., Ltd. (Harbin, China), Tailong Pharmaceutical Co., Ltd. (Zhengzhou, China), Zhenbaodao Pharmaceutical Co., Ltd. (Harbin, China) and Baitian'e Pharmaceutical Co., Ltd. (Harbin, China), respectively. All sample solutions were ultrasonically extracted with methanol-water $(50: 50, v / v)$ for $20 \mathrm{~min}$. The sample solutions were filtered through a $0.22 \mu \mathrm{m}$ membrane filter before it was injected into the UPLC system for analysis.

\subsection{Chromatographic and MS Conditions}

Analysis was performed using an ACQUITY UPLC system with a conditioned autosampler at $4{ }^{\circ} \mathrm{C}$. Chromatographic separation was carried out at $35^{\circ} \mathrm{C}$ on an ACQUITY UPLC HSS T3 column $(1.8 \mu \mathrm{m}, 2.1 \mathrm{~mm} \times 100 \mathrm{~mm})$. The mobile phase was composed of $\mathrm{A}(0.1 \%$ formic acid in water $)$ and $\mathrm{B}$ (0.1\% formic acid in acetonitrile) with a gradient elution: $0-5 \mathrm{~min}, 87-84 \%(\mathrm{~A}) ; 5-7 \mathrm{~min}, 84-80 \%(\mathrm{~A})$; 7-15 $\mathrm{min}, 80-78 \%(\mathrm{~A})$ and $15-23 \mathrm{~min}, 78-70 \%(\mathrm{~A})$. The flow rate of the mobile phase was $0.2 \mathrm{~mL} / \mathrm{min}$, and the injection volume was $2 \mu \mathrm{L}$.

The mass spectrometry was performed on a 4000 QTRAP LC-MS/MS system (AB Sciex, Foster City, CA, USA) equipped with ESI interface in negative mode. All instruments were controlled and synchronized by Analyst software (version 1.6, SCIEX, AB Sciex, Foster City, CA, USA). Ion spray voltage was set at (3300) $\mathrm{V}$, turbo spray temperature was $550{ }^{\circ} \mathrm{C}$ and interface heater was on. Both nebulizer gas (gas 1) and heater gas (gas 2) were set at 55 psi.

\section{Conclusions}

In this study, a new UPLC-ESI ${ }^{-}$-MRM-MS/MS method has been developed for the simultaneous determination of 18 major components in SHL oral liquid. This method was advantaged for rapid and simultaneous determination of multiple classes of hydrophilic and lipophilic components in SHL oral 
liquids by comparison with previous reports. These compounds include two quinic acids (1 and 11), one phenylpropionic acid (2), three phenylethanoid glycosides (3, 4 and 7), eight flavonoids (5, 6, $8,9,12,13,17$ and 18), four lignans (10 and 14-16). This novel evaluation approach can overcome the deficiencies of previously described methods revealing the complexity of samples from the same or different manufacturers. It provides much more qualitative information than any other singular evaluation. Data analysis on the $14 \mathrm{SHL}$ oral liquid samples suggested that the concentration of the some compounds varied significantly from different manufacturers of China. The proposed method had been elucidated to be a simple, sensitive, accurate and reliable quality control procedure of SHL oral liquids.

Supplementary Materials: Supplementary Materials are available online.

Acknowledgments: This study was partly financially supported by University Nursing Program for Young Scholars with Creative Talents in Heilongjiang Province (2016209).

Author Contributions: J.L. was planned and executed the laboratory experiments. H.-M.S. and T.-L.W. participated in data analysis and results interpretation. All authors read and approved the final manuscript.

Conflicts of Interest: The authors declare no conflict of interest.

\section{References}

1. Ye, J.X.; Wei, W.; Quan, L.H.; Liu, C.Y.; Chang, Q.; Liao, Y.H. An LC-MS/MS method for the simultaneous determination of chlorogenic acid, forsythiaside $\mathrm{A}$ and baicalin in rat plasma and its application to pharmacokinetic study of Shuang-huang-lian in rats. J. Pharm. Biomed. Anal. 2010, 52, 625-630. [CrossRef] [PubMed]

2. Ma, Y.C.; Wang, X.Q.; Hou, F.F.; Ma, J.; Luo, M.; Chen, A.; Jin, P.; Lu, S.; Xu, I. Rapid resolution liquid chromatography (RRLC) analysis and studies on the stability of Shuang-Huang-Lian preparations. J. Pharm. Biomed. Anal. 2011, 54, 265-272. [CrossRef] [PubMed]

3. Cao, Y.; Wang, L.; Yu, X. Development of the chromatographic fingerprint of herbal preparations Shuang-Huang-Lian oral liquid. J. Pharm. Biomed. Anal. 2006, 41, 845-856. [CrossRef] [PubMed]

4. Yan, G.L.; Zhang, A.H.; Sun, H. An effective method for determining the ingredients of Shuanghuanglian formula in blood samples using high-resolution LC-MS coupled with background subtraction and a multiple data processing approach. J. Sep. Sci. 2013, 36, 3191-3199. [CrossRef] [PubMed]

5. Chen, G.; Zhang, H.W.; Ye, J.N. Determination of baicalein, baicalin and quercetin in Scutellariae Radix and its preparations by capillary electrophoresis with electrochemical detection. Talanta 2000, 53, 471-479. [CrossRef]

6. Yang, L.I.; Yan, Q.; Mo, H.Z. Determination of chlorogenic acid, baicalin and forsythin in Shuanghuanglian preparations by HPLC-DAD. J. Chilean Chem. Soc. 2012, 57, 1361-1363. [CrossRef]

7. Ban, L.N.; Xu, Y.J. Simultaneous determination of four effective components in Shuanghuanglian oral liquid by HPLC/MS. Chin. Tradit. Pat. Med. 2012, 34, 265-268.

8. Zhang, Y.M.; Yan, D.; Zhang, P.; Ren, Y.S.; Zhang, S.F.; Feng, X.; Xiao, X.H. Quality control of Shuanghuanglian freeze-dried powder for injection based on its HPLC-ELSD fingerprints and biological profiles. Acta Pharm. Sin. 2010, 45, 93-97.

9. Liu, L.; Suo, Z.R.; Zheng, J.B. Simultaneous determination of four compounds in Sanjing Shuanghuanglian oral liquid by high performance liquid chromatography-diode array detection-electrochemical detection. Chin. J. Chromatogr. 2006, 24, 247-250.

10. Liu, T.; Di, L.Q.; Peng, L.X.; Kang, A.; Li, J.S.; Zhao, X.L. Simultaneous determination of 17 major components of Shuang-Huang-Lian oral liquid by UPLC-MS/MS. Chin. Tradit. Herb. Drugs 2015, 46, 3357-3363.

11. Huang, M.; Zhao, H.; Xu, W.; Chu, K.; Hong, Z.; Peng, J.; Chen, L. Rapid simultaneous determination of twelve major components in Pien Tze Huang by ultra-performance liquid chromatography coupled with triple quadrupole mass spectrometry. J. Sep. Sci. 2013, 36, 3866-3873. [CrossRef] [PubMed]

12. Qian, Z.M.; Li, H.J.; Li, P. Simultaneous qualitation and quantification of thirteen bioactive compounds in Flos lonicerae by high-performance liquid chromatography with diode array detector and mass spectrometry. Chem. Pharm. Bull. 2007, 55, 1073-1076. [CrossRef] [PubMed] 
13. Zuo, F.; Zhou, Z.M.; Liu, M.L. Determination of 14 chemical constituents in the traditional Chinese medicinal preparation Huangqin-Tang by high performance liquid chromatography. Biol. Pharm. Bull. 2001, 24, 693-697. [CrossRef] [PubMed]

14. Guo, H.; Liu, A.H.; Li, L. Simultaneous determination of 12 major constituents in Forsythia suspensa by high performance liquid chromatography-DAD method. J. Pharm. Biomed. Anal. 2007, 43, 1000-1006. [CrossRef] [PubMed]

Sample Availability: Not available.

(C) 2017 by the authors. Licensee MDPI, Basel, Switzerland. This article is an open access article distributed under the terms and conditions of the Creative Commons Attribution (CC BY) license (http:/ / creativecommons.org/licenses/by/4.0/). 